\title{
CENTRAL SIMPLE ALGEBRAS WITH INVOLUTION: A GEOMETRIC APPROACH
}

\author{
NIKOLAUS VONESSEN
}

\begin{abstract}
Let $k$ be an algebraically closed base field of characteristic zero. The category equivalence between central simple algebras and irreducible, generically free $\mathrm{PGL}_{n}$-varieties is extended to the context of central simple algebras with involution. The associated variety of a central simple algebra with involution comes with an action of $\mathrm{PGL}_{n} \rtimes\langle\tau\rangle$, where $\tau$ is the automorphism of $\mathrm{PGL}_{n}$ given by $\tau(h)=\left(h^{-1}\right)^{\text {transpose }}$. Basic properties of an involution are described in terms of the action of $\mathrm{PGL}_{n} \rtimes\langle\tau\rangle$ on the associated variety, and in particular in terms of the stabilizer in general position for this action.
\end{abstract}

\section{Contents}

1. Introduction

2. A Category Equivalence

3. Involutions of the First and Second Kind

4. Preliminaries on Transposition in $\mathrm{PGL}_{n}$

5. Proof of Theorem 1.2

6. The Involution $\rho$ of $U D(2,2)$

7. Models for Associated Varieties

References

\section{INTRODUCTION}

We work over an algebraically closed base field $k$ of characteristic zero. In this paper, a central simple algebra is a simple algebra finite-dimensional over its center with the additional property that the center is a finitelygenerated field extension of $k$. We begin by reviewing some facts, mostly from [10], about central simple algebras and their associated varieties. Given an irreducible, generically free $\mathrm{PGL}_{n}$-variety $X, k_{n}(X)$ denotes the set of $\mathrm{PGL}_{n}$-equivariant rational maps from $X$ to $\mathrm{M}_{n}$, the algebra of $n \times n$ matrices over $k$. Under the usual addition and multiplication of maps into $\mathrm{M}_{n}, k_{n}(X)$

Date: August 12, 2008.

2000 Mathematics Subject Classification. 16W10, 14L30, 16K20.

Key words and phrases. Central simple algebra with involution, associated variety, group action, stabilizer in general position, rational quotient. 
turns out to be a central simple algebra; its center can be identified with the fixed field $k(X)^{\mathrm{PGL}_{n}}$ ([8, Lemma 8.5]; see also [11, Lemma 2.8]).

Conversely, let $A$ be a central simple algebra (whose center is, by our general convention, a finitely-generated field extension of $k$ ). Then there is an irreducible, generically free $\mathrm{PGL}_{n}$-variety $X$ such that $A$ is isomorphic to $k_{n}(X)$. In fact, $X$ is unique up to birational isomorphism of $\mathrm{PGL}_{n}$-varieties, and it is called the $\mathrm{PGL}_{n}$-variety associated to $A$ ( $\sqrt{10}$, Theorem 7.8 and Section 8]).

Now let $A$ and $B$ be central simple algebras with associated $\mathrm{PGL}_{n^{-}}$ varieties $X$ and $Y$, respectively. A $\mathrm{PGL}_{n}$-equivariant dominant rational map $\varphi: X \rightarrow Y$ induces an algebra homomorphism (necessarily an embedding) $\varphi^{*}: k_{n}(Y) \hookrightarrow k_{n}(X)$ defined by $f \mapsto f \circ \varphi$. Conversely, an algebra homomorphism $\alpha: k_{n}(Y) \hookrightarrow k_{n}(X)$ induces a $\mathrm{PGL}_{n}$-equivariant dominant rational map $\alpha_{*}: X \rightarrow Y$. The expected functorial properties hold: $\left(\varphi^{*}\right)_{*}=\varphi$, $\left(\alpha_{*}\right)^{*}=\alpha,\left(\operatorname{id}_{k_{n}(X)}\right)_{*}=\operatorname{id}_{X},\left(\operatorname{id}_{X}\right)^{*}=\operatorname{id}_{k_{n}(X)},(\psi \circ \varphi)^{*}=\varphi^{*} \circ \psi^{*}$, and $(\beta \circ \alpha)_{*}=\alpha_{*} \circ \beta_{*}([10$, Section 8]). In fact, the functor defined by

$$
X \mapsto k_{n}(X), \quad(\varphi: X \rightarrow Y) \mapsto\left(\varphi^{*}: k_{n}(Y) \hookrightarrow k_{n}(X)\right)
$$

is a contravariant equivalence of categories from the category $B_{i} r_{n}$ of irreducible, generically free $\mathrm{PGL}_{n}$-varieties (with dominant rational $\mathrm{PGL}_{n}$ equivariant maps as morphisms) to the category $C S_{n}$ of central simple algebras of degree $n$ (with algebra homomorphisms as morphisms) ( 10 , Theorem 1.2]).

The Results. We denote by $\tau$ the automorphism of order two of $\mathrm{PGL}_{n}$ defined by

$$
\tau(h)=\left(h^{-1}\right)^{t}=\left(h^{t}\right)^{-1},
$$

where $t$ denotes transposition. Central for our work is the semidirect product

$$
P_{n, \tau}:=\mathrm{PGL}_{n} \rtimes\langle\tau\rangle \text {. }
$$

Throughout, we identify $\mathrm{PGL}_{n}$ with the subgroup $\mathrm{PGL}_{n} \times 1$ of $P_{n, \tau}$.

Given a $P_{n, \tau}$-variety $X$ which is $\mathrm{PGL}_{n}$-generically free, the central simple algebra $k_{n}(X)$ has an involution $\sigma_{X, \tau}$ induced by the action of $\tau$ on $X$ : For $f \in k_{n}(X)$,

$$
\sigma_{X, \tau}(f)=\text { transposition } \circ f \circ \tau,
$$

see Lemma 2.1. Conversely, given a central simple algebra $A$ of degree $n$ with involution $\sigma$, there is an irreducible $P_{n, \tau^{-}}$ariety $X$ which is $\mathrm{PGL}_{n^{-}}$ generically free such that $(A, \sigma)$ and $\left(k_{n}(X), \sigma_{X, \tau}\right)$ are isomorphic as algebras with involution (Proposition 2.3). Moreover, $X$ is unique up to birational isomorphism of $P_{n, \tau}$-varieties (Corollary 2.6). We call $X$ the $P_{n, \tau}$-variety associated to $(A, \sigma)$. A model for $X$ can usually be found in $\left(\mathrm{M}_{n}\right)^{m}$ for some $m$ (unless $n=2$ and the involution is symplectic), see Lemma 7.1.

The category equivalence between $B_{i r}$ and $C S_{n}$ extends to this setting. In the sequel, the morphisms in categories of algebras with involution are the $k$-algebra homomorphisms preserving involutions. And on the geometric 
side, the morphisms are the dominant rational maps which are equivariant for the action of the given group.

Theorem 1.1. There is a contravariant category equivalence

$$
\begin{aligned}
& \text { Irreducible } P_{n, \tau} \text {-varieties } \\
& \text { which are generically free } \\
& \text { as } \mathrm{PGL}_{n} \text {-varieties } \\
& \text { Central simple algebras of } \\
& \text { degree } n \text { with involution }
\end{aligned}
$$

given by the contravariant functor

$$
\begin{array}{ccc}
X & \longmapsto & \left(k_{n}(X), \sigma_{X, \tau}\right) \\
(\varphi: X \rightarrow Y) & \longmapsto & \left(\varphi^{*}: k_{n}(Y) \hookrightarrow k_{n}(X)\right) .
\end{array}
$$

This result is proved at the end of Section 2. We show next how basic properties of an involution of a central simple algebra are related to properties of the $P_{n, \tau}$-action on the associated variety. Recall that an action of a linear algebraic group $G$ on a variety $X$ has a stabilizer in general position if there is a subgroup $S$ of $G$ such that for $x \in X$ in general position, the stabilizer of $x$ in $G$ is conjugate to $S$.

Theorem 1.2. Let $A$ be a central simple algebra of degree $n$ with involution $\sigma$ and associated $P_{n, \tau}$-variety $X$. Then the $P_{n, \tau}$-action on $X$ has a stabilizer in general position, say $S$.

(a) The involution $\sigma$ is of the first kind iff $S \neq 1$.

(b) The involution $\sigma$ is of the second kind (i.e, is unitary) iff $S=1$ (i.e., the action of $P_{n, \tau}$ on $X$ is generically free).

(c) The involution $\sigma$ is orthogonal iff $S$ is conjugate to $\{1, \tau\}$.

(d) The involution $\sigma$ is symplectic iff $S$ is conjugate to $\left\{1, \tau\left[\begin{array}{cc}0 & I \\ -I & 0\end{array}\right]\right\}$.

In part $(\mathrm{d}), g_{0}=\left[\begin{array}{cc}0 & I \\ -I & 0\end{array}\right]$ denotes the image in $\mathrm{PGL}_{n}$ of the matrix $\left(\begin{array}{cc}0 & I \\ -I & 0\end{array}\right)$, where $I$ is the identity matrix of order $\frac{n}{2}$. Note that since $S$ is only defined up to conjugacy, one can always take it to be $\{1, \tau\}$ if $\sigma$ is orthogonal, and to be $\left\{1, \tau g_{0}\right\}$ if $\sigma$ is symplectic.

This theorem is proved in Section 5. The existence of the stabilizer in general position follows easily from a deep theorem of Richardson (see Remark (3.4) but will be deduced independently from the two well-known facts that all symmetric matrices in $\mathrm{M}_{n}$ are congruent to each other, and that all skew-symmetric matrices in $\mathrm{M}_{n}$ are congruent to each other. These facts are also otherwise needed in the proof of Theorem 1.2, and this approach makes its proof only marginally longer.

We conclude with two remarks.

Remark 1.3. Let $\sigma$ be an involution of the first kind. Whether $\sigma$ is orthogonal or symplectic can also be described in the following, more geometric fashion: The proof of Theorem 1.2 in Section 5 shows that in the case of involutions of the first kind, for all $x \in X$ in general position, there is a unique $g_{x} \in \mathrm{PGL}_{n}$ such that $\tau(x)=g_{x}(x)$; moreover, there are two mutually exclusive cases: either the preimages of $g_{x}$ in $\mathrm{GL}_{n}$ are symmetric matrices for all 
$x \in X$ in general position, or they are skew-symmetric matrices for all $x \in X$ in general position (cf. Lemma 5.1). Accordingly, we say that the action of $\tau$ on $X$ is of symmetric or skew-symmetric type. Finally, $\sigma$ is orthogonal if and only if the action of $\tau$ on $X$ is of symmetric type, and $\sigma$ is symplectic if and only if the action of $\tau$ on $X$ is of skew-symmetric type (see Lemma 5.1). See Section 6 for an example.

Remark 1.4. Theorems 1.1 and 1.2 can be used to give a new proof of the following well-known contravariant category equivalences (see[3, §29] for similar results under less restrictive hypotheses):

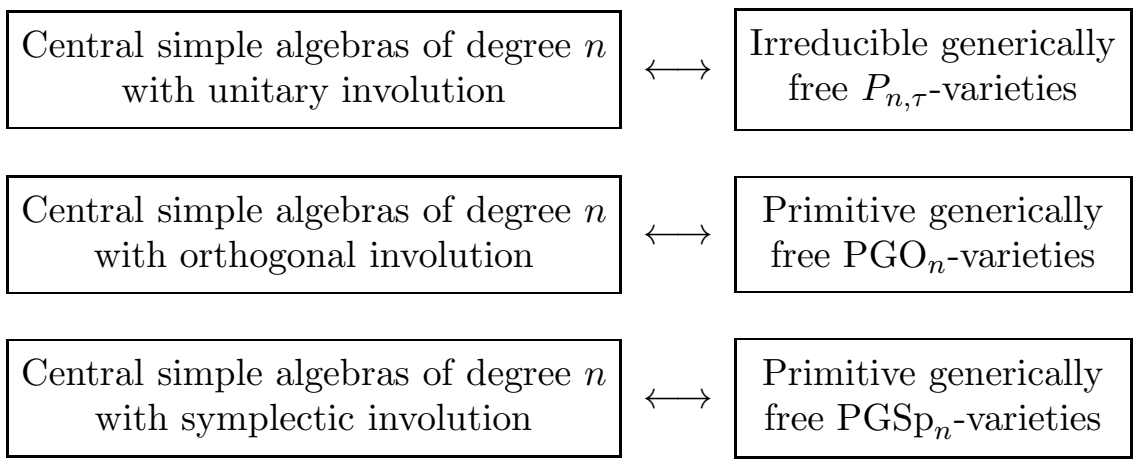

The first equivalence follows immediately from Theorems 1.1 and 1.2. For the others, one needs in addition the following fact: Let $G$ be a linear algebraic group, $S$ a closed subgroup and $N$ the normalizer of $S$ in $G$. There is a category equivalence between the category of generically free primitive $N / S$-varieties and the category of primitive $G$-varieties with stabilizer $S$ in general position, cf. [4], or [9, Lemma 3.2] (which in turn is based on [5, Section 1.7]). Note that if $G=P_{n, \tau}$ and $S$ is equal to $\langle\tau\rangle$ or $\left\langle\tau g_{0}\right\rangle$, then $N / S$ is isomorphic to $\mathrm{PGO}_{n}$ or $\mathrm{PGSp}_{n}$, respectively, as easy computations show.

Conventions. As already stated, $k$ is an algebraically closed base field of characteristic zero, and a central simple algebra is a simple algebra finitedimensional over its center with the additional property that the center is a finitely-generated field extension of $k$. All algebra homomorphisms and involutions are assumed to be $k$-linear. We always denote by $m$ and $n$ integers $\geq 2$. We write $\mathrm{M}_{n}$ for $\mathrm{M}_{n}(k), \mathrm{GL}_{n}$ for $\mathrm{GL}_{n}(k), \mathrm{PGL}_{n}$ for $\mathrm{PGL}_{n}(k)$, etc. The transpose of a matrix $a$ is denoted by $a^{t}$. If $a=\left(a_{1}, \ldots, a_{m}\right)$ is an $m$-tuple of matrices, then $a^{t}=\left(a_{1}^{t}, \ldots, a_{m}^{t}\right)$.

Acknowledgments. The author is grateful to Zinovy Reichstein for helpful comments and suggestions.

\section{A Category Equivalence}

The generic matrix ring

$$
G_{m, n}=k\left\{X_{1}, \ldots, X_{m}\right\}
$$


has an involution $\rho$ which is the $k$-linear map which reverses the order of any monomial in the $m$ generic $n \times n$-matrices $X_{1}, \ldots, X_{m}$. That is,

$$
\rho\left(X_{i_{1}} X_{i_{2}} \cdots X_{i_{j}}\right)=X_{i_{j}} \cdots X_{i_{2}} X_{i_{1}} .
$$

To see that $\rho$ is well-defined, consider the correspondingly defined map $\rho_{F}$ on the free algebra $F=k\left\{x_{1}, \ldots, x_{m}\right\}$. It suffices to show that if $p=$ $p\left(x_{1}, \ldots, x_{m}\right) \in F$ is a polynomial identity for $n \times n$-matrices, then so is $\rho_{F}(p)$. Well, let $a=\left(a_{1}, \ldots, a_{m}\right)$ be any $m$-tuple of $n \times n$-matrices. Then

$$
\rho_{F}(p)\left(a_{1}^{t}, \ldots, a_{m}^{t}\right)=p\left(a_{1}, \ldots, a_{m}\right)^{t} .
$$

That is,

$$
\rho_{F}(p)\left(a_{1}, \ldots, a_{m}\right)=p\left(a_{1}^{t}, \ldots, a_{m}^{t}\right)^{t}=0 .
$$

The involution $\rho$ of $G_{m, n}$ is induced from transposition of $m$-tuples of $n \times n$ matrices in the sense that for any $p=p\left(X_{1}, \ldots, X_{m}\right) \in G_{m, n}$,

$$
\rho(p)\left(a_{1}, \ldots, a_{m}\right)=p\left(a_{1}^{t}, \ldots, a_{m}^{t}\right)^{t},
$$

or

$$
\rho(p)(a)=p\left(a^{t}\right)^{t}
$$

Lemma 2.1. Let $X$ be an irreducible $P_{n, \tau}$-variety which is $\mathrm{PGL}_{n}$-generically free, so that $A=k_{n}(X)$ is a central simple algebra of degree $n$. Then the action of $\tau$ on $X$ induces an involution $\sigma_{X, \tau}$ of $A$ as follows: For $f \in A$,

$$
\sigma_{X, \tau}(f)=\text { transposition } \circ f \circ \tau \text {. }
$$

That is, for $x \in X$ in general position,

$$
\sigma_{X, \tau}(f)(x)=f(\tau x)^{t} .
$$

Note the similarity of formulas (2.2) and (2.3).

Proof. For simplicity, set $\sigma=\sigma_{X, \tau}$. It is clear that $\sigma(f)$ is a rational map $X \rightarrow \mathrm{M}_{n}$. To check that it is $\mathrm{PGL}_{n}$-equivariant, let $h \in \mathrm{PGL}_{n}$ and $f \in A$. Keeping in mind that $\mathrm{PGL}_{n}$ acts on $\mathrm{M}_{n}$ by conjugation, and that $f$ is $\mathrm{PGL}_{n^{-}}$ equivariant, one sees that for $x \in X$ in general position,

$$
\begin{aligned}
\sigma(f)(h x) & =f(\tau h x)^{t}=\left[f\left(\left(h^{-1}\right)^{t} \tau x\right)\right]^{t}=\left[\left(h^{-1}\right)^{t} f(\tau x) h^{t}\right]^{t} \\
& =h f(\tau x)^{t} h^{-1}=h[\sigma(f)(x)] h^{-1} .
\end{aligned}
$$

That is, $\sigma(f)$ is $\mathrm{PGL}_{n}$-equivariant and belongs thus to $A$.

It is clear that $\sigma^{2}$ is the identity map of $A$, and that $\sigma$ is $k$-linear. Finally, let $f, g \in A$. Then for $x \in X$ in general position,

$$
\sigma(f g)(x)=[(f g)(\tau x)]^{t}=g(\tau x)^{t} \cdot f(\tau x)^{t}=[\sigma(g) \sigma(f)](x) .
$$

So $\sigma(f g)=\sigma(g) \sigma(f)$, and $\sigma$ is indeed an involution of $A$. 
Example 2.2. The involution $\rho$ of $U D(m, n)$. The universal division algebra $U D(m, n)$ is obtained from $G_{m, n}$ by localizing at the non-zero central elements. It can also be obtained from $G_{m, n}$ by inverting only the nonzero central symmetric elements (since for $p, s \in G_{m, n}$ with $s$ nonzero and central, $\left.p s^{-1}=(p \rho(s))(s \rho(s))^{-1}\right)$. Hence the involution $\rho$ of $G_{m, n}$ extends to an involution of $U D(m, n)$, which we will also denote by $\rho$ (cf. 13, Proposition 2.13.17]). One checks easily that for all $p, s \in G_{m, n}$ with $s$ nonzero and central, $\rho\left(p s^{-1}\right)=\rho(p) \rho(s)^{-1}$.

The associated variety of $U D(m, n)$ is $X=\left(\mathrm{M}_{n}\right)^{m}$, where $\mathrm{PGL}_{n}$ acts by simultaneous conjugation. Letting $\tau$ act by simultaneous transposition turns $X=\left(\mathrm{M}_{n}\right)^{m}$ into a $P_{n, \tau}$-variety which is $\mathrm{PGL}_{n}$-generically free. Formula (2.2) says that for all $p \in G_{m, n}$ and all $x \in X, \rho(p)(x)=p(\tau x)^{t}=$ $\sigma_{X, \tau}(x)$. A straightforward calculation shows that for all $f \in U D(m, n)$, $\rho(f)(x)=\sigma_{X, \tau}(x)$ for all $x \in X$ in general position. Hence $\rho=\sigma_{X, \tau}$. We will return to this example in Example 3.5 and in Section 6.

Proposition 2.3. Let $A$ be a central simple algebra of degree $n$ with involution $\sigma$. As always, we assume that the center of $A$ is a finitely generated field extension of $k$. Then there is an irreducible $P_{n, \tau}$-variety $X$ which is $\mathrm{PGL}_{n}$-generically free such that $(A, \sigma)$ and $\left(k_{n}(X), \sigma_{X, \tau}\right)$ are isomorphic as algebras with involution.

Here $\sigma_{X, \tau}$ is the involution defined in Lemma 2.1. We call $X$ the $P_{n, \tau^{-}}$ variety associated to $(A, \sigma)$. We will see below that $X$ is unique up to birational isomorphism of $P_{n, \tau}$-varieties (Corollary 2.6). Lemma 7.1 shows that up to birational isomorphism, a model for $X$ can in most cases be found in $\left(\mathrm{M}_{n}\right)^{m}$.

Proof. There is a finite-dimensional, $\sigma$-stable $k$-subspace $V$ of $A$ such that the $k$-algebra $R$ generated by $V$ is prime, and such that $A$ is the total ring of fractions of $R$. Choose a basis $r_{1}, \ldots, r_{m}$ of $V$ consisting of eigenvectors of $\sigma$. More precisely, $\sigma\left(r_{i}\right)=\epsilon_{i} r_{i}$ where $\epsilon_{i}= \pm 1$. Note that $R=k\left\{r_{1}, \ldots, r_{m}\right\}$.

Consider the homomorphism $\phi: G_{m, n} \longrightarrow R$ defined by $X_{i} \mapsto r_{i}$. Denote its kernel by $I$. Recall that $\mathcal{Z}(I)$ is the set of common zeroes of the elements of $I$ among the points in a certain $\mathrm{PGL}_{n}$-stable dense open subset of $\left(\mathrm{M}_{n}\right)^{m}$, see [10, Definition 3.1]. Set $X=\mathcal{Z}(I)$. Then by construction, $X$ is an associated variety of $A$, see 10, Theorems 6.4 and 7.8]. Moreover, $\phi$ induces $k$-algebra isomorphisms from $G_{m, n} / I$ onto $R$, and from $\mathrm{Q}\left(G_{m, n} / I\right)=k_{n}(X)$ onto $A$. We will denote both of these isomorphisms by $\bar{\phi}$.

We first lift $\sigma$ to an involution $\tilde{\sigma}$ of $G_{m, n}$. For $p\left(X_{1}, \ldots, X_{m}\right) \in G_{m, n}$, define

$$
\tilde{\sigma}(p)=\rho\left(p\left(\epsilon_{1} X_{1}, \ldots, e_{m} X_{m}\right)\right),
$$

where $\rho$ is the involution of $G_{m, n}$ defined in (2.1). Using that the assignment $X_{i} \mapsto \epsilon_{i} X_{i}$ defines an automorphism of $G_{m, n}$, one easily checks that $\tilde{\sigma}$ is 
indeed an involution of $G_{m, n}$. Moreover,

$$
\begin{aligned}
\phi\left(p\left(\epsilon_{1} X_{1}, \ldots, \epsilon_{m} X_{m}\right)\right) & =p\left(\epsilon_{1} r_{1}, \ldots, \epsilon_{m} r_{m}\right) \\
& =p\left(\sigma\left(r_{1}\right), \ldots, \sigma\left(r_{m}\right)\right)=\sigma(\phi(\rho(p))) .
\end{aligned}
$$

Replacing $p$ by $\rho(p)$, one sees that

$$
\phi \circ \tilde{\sigma}=\sigma \circ \phi .
$$

In particular, the kernel $I$ of $\phi$ is $\tilde{\sigma}$-stable. Thus the involution $\tilde{\sigma}$ of $G_{m, n}$ induces involutions $\overline{\tilde{\sigma}}$ of $G_{m, n} / I$ and of $\mathrm{Q}\left(G_{m, n} / I\right)=k_{n}(X)$. Since $\phi \circ \tilde{\sigma}=$ $\sigma \circ \phi$, the $k$-algebra isomorphism $\bar{\phi}: k_{n}(X) \longrightarrow A$ is actually an isomorphism of central simple algebras with involution. In order to prove the proposition, we may thus assume that $A=k_{n}(X)$, that $R=G_{m, n} / I$, and that the image of the generic matrix $X_{i}$ in $R$ is $r_{i}$. So $\phi$ is now the natural projection from $G_{m, n}$ onto $R=G_{m, n} / I$, and $\overline{\tilde{\sigma}}=\sigma$.

We will now make $X=\mathcal{Z}(I)$ into a $P_{n, \tau}$-variety, and show that the involution $\sigma$ on $A=k_{n}(X)$ is equal to $\sigma_{X, \tau}$.

We first define an action of $\tau$ on $\left(\mathrm{M}_{n}\right)^{m}$ as follows:

$$
\tau\left(a_{1}, \ldots, a_{m}\right)=\left(\epsilon_{1} a_{1}^{t}, \ldots, \epsilon_{m} a_{m}^{t}\right)
$$

Then for $p \in G_{m, n}$ and $a=\left(a_{1}, \ldots, a_{m}\right) \in\left(\mathrm{M}_{n}\right)^{m}$,

$$
\begin{aligned}
p(\tau a) & =p\left(\epsilon_{1} a_{1}^{t}, \ldots, \epsilon_{m} a_{m}^{t}\right) \\
& =\left(\left[\rho\left(p\left(\epsilon_{1} X_{1}, \ldots, \epsilon_{m} X_{m}\right)\right)\right]\left(a_{1}, \ldots, a_{m}\right)\right)^{t} \\
& =(\tilde{\sigma}(p)(a))^{t} .
\end{aligned}
$$

This shows that

$$
\tilde{\sigma}(p)(a)=p(\tau a)^{t} .
$$

It also shows that if $p \in I$ and $a \in X=\mathcal{Z}(I)$, then $p(\tau a)=0$ (since $\tilde{\sigma}(p) \in I)$. Hence $\tau(a) \in X$. Consequently, $\tau(X)=X$.

We verify next that $\left(\mathrm{M}_{n}\right)^{m}$ is a $P_{n, \tau}$-variety. To show this, it suffices to check that for $h \in \mathrm{PGL}_{n}$ and $a=\left(a_{1}, \ldots, a_{m}\right) \in\left(\mathrm{M}_{n}\right)^{m}$,

$$
[\tau h](a)=\left[\left(h^{-1}\right)^{t} \tau\right](a) .
$$

Well,

$$
\begin{aligned}
{[\tau h](a) } & =\tau\left(h a_{1} h^{-1}, \ldots, h a_{m} h^{-1}\right)=\left(\epsilon_{1}\left(h a_{1} h^{-1}\right)^{t}, \ldots, \epsilon_{m}\left(h a_{m} h^{-1}\right)^{t}\right) \\
& =\left(\left(h^{-1}\right)^{t}\left(\epsilon_{1} a_{1}^{t}\right) h^{t}, \ldots,\left(h^{-1}\right)^{t}\left(\epsilon_{m} a_{m}^{t}\right) h^{t}\right)=\left[\left(h^{-1}\right)^{t} \tau\right](a) .
\end{aligned}
$$

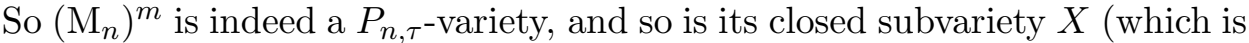
both $\mathrm{PGL}_{n^{-}}$and $\langle\tau\rangle$-stable). In particular, the action of $\tau$ on $X$ defines now the involution $\sigma_{X, \tau}$ on $A=k_{n}(X)$. It only remains to show that $\sigma=\sigma_{X, \tau}$, i.e., that for all $f \in A$ and all $a \in X$ in general position,

$$
\sigma(f)(a)=f(\tau a)^{t},
$$


cf. Lemma 2.1. It suffices to verify this for $f \in R=G_{m, n} / I$. So let $p \in G_{m, n}$ such that $f=\phi(p)$. Then by definition, $f(a)=p(a)$ for all $a \in X=\mathcal{Z}(I)$. Hence

$$
\begin{gathered}
\sigma(f)(a)=[\sigma(\phi(p))](a) \stackrel{(2.4)}{=}[\phi(\tilde{\sigma}(p))](a)=\tilde{\sigma}(p)(a) \\
\stackrel{2.5)}{=} p(\tau a)^{t}=[\phi(p)(\tau a)]^{t}=f(\tau a)^{t} .
\end{gathered}
$$

Lemma 2.4. Let $X$ and $Y$ be irreducible $P_{n, \tau}$-varieties which are $\mathrm{PGL}_{n}$ generically free. Denote by $\sigma_{X, \tau}$ and $\sigma_{Y, \tau}$ the involutions of $k_{n}(X)$ and $k_{n}(Y)$ induced by the actions of $\tau$ on $X$ and $Y$, respectively.

(a) Let $\varphi: X \rightarrow Y$ be a $P_{n, \tau}$-equivariant dominant rational map. Then the induced $k$-algebra homomorphism $\varphi^{*}: k_{n}(Y) \longrightarrow k_{n}(X)$ is a homomorphism of algebras with involution.

(b) Let $\alpha: k_{n}(Y) \longrightarrow k_{n}(X)$ be a homomorphism of algebras with involution. Then the induced $\mathrm{PGL}_{n}$-equivariant dominant rational map $\alpha_{*}: X \rightarrow Y$ is $P_{n, \tau}$-equivariant.

Proof. (a) Let $f \in k_{n}(Y)$. We have to show that $\varphi^{*}\left(\sigma_{Y, \tau}(f)\right)=\sigma_{X, \tau}\left(\varphi^{*}(f)\right)$. By definition, $\varphi^{*}(f)=f \circ \varphi$. So we have to show that

$$
\sigma_{Y, \tau}(f) \circ \varphi=\sigma_{X, \tau}(f \circ \varphi) .
$$

By Lemma 2.1, this reduces to showing that

$$
\left(f \circ \tau_{Y}\right) \circ \varphi=(f \circ \varphi) \circ \tau_{X},
$$

where $\tau_{X}$ and $\tau_{Y}$ denote the actions of $\tau$ on $X$ and $Y$, respectively. But this last equation is true since we are assuming that $\varphi$ is $\langle\tau\rangle$-equivariant.

(b) We know that $\varphi=\alpha_{*}$ is $\mathrm{PGL}_{n}$-equivariant. So it suffices to show that $\tau_{Y} \circ \varphi=\varphi \circ \tau_{X}$. Note that $\varphi^{*}=\alpha$. Let $f \in k_{n}(Y)$. We are assuming that $\varphi^{*}\left(\sigma_{Y, \tau}(f)\right)=\sigma_{X, \tau}\left(\varphi^{*}(f)\right)$. Arguing as in part (a), we deduce from this that

$$
f \circ\left(\tau_{Y} \circ \varphi\right)=f \circ\left(\varphi \circ \tau_{X}\right) \quad \text { for all } f \in k_{n}(Y) \text {. }
$$

By the next lemma, this implies that $\tau_{Y} \circ \varphi=\varphi \circ \tau_{X}$.

Lemma 2.5. Let $Y$ be an irreducible generically free $\mathrm{PGL}_{n}$-variety, and $X$ any variety. Let $\varphi_{1}$ and $\varphi_{2}$ be two dominant rational maps from $X$ to $Y$ such that $f \circ \varphi_{1}=f \circ \varphi_{2}$ for all $f \in k_{n}(Y)$. Then $\varphi_{1}=\varphi_{2}$.

Proof. By [10, Lemma 8.1], $Y$ is birationally isomorphic (as $\mathrm{PGL}_{n}$-variety) to a $\mathrm{PGL}_{n}$-subvariety $Y^{\prime}$ of $\left(\mathrm{M}_{n}\right)^{m}$ for some integer $m \geq 2$. Thus we may assume $Y=Y^{\prime}$. Substituting $f=p_{i}$ for $i=1, \ldots, m$, where $p_{i}: Y \longrightarrow \mathrm{M}_{n}$ is the projection from $Y \subseteq\left(\mathrm{M}_{n}\right)^{m}$ to the $i$ th copy of $\mathrm{M}_{n}$, we see that $\varphi_{1}=\varphi_{2}$.

Corollary 2.6. The associated $P_{n, \tau}$-variety of a central simple algebra with involution is unique up to birational isomorphism of $P_{n, \tau}$-varieties. 
Proof. Let $(A, \sigma)$ and $X$ be as in Proposition 2.3. Say $Y$ is another associated $P_{n, \tau}$-variety of $(A, \sigma)$. Then there is an isomorphism of algebras with involution $\alpha: k_{n}(Y) \longrightarrow k_{n}(X)$. By [10, Corollary 8.6], the induced $\mathrm{PGL}_{n^{-}}$ equivariant dominant rational map $\alpha_{*}: X \rightarrow Y$ is a birational isomorphism, and it is $P_{n, \tau}$-equivariant by Lemma 2.4 .

The following proof is modeled after the proof of [10, Theorem 1.2].

Proof of Theorem 1.1. Denote by $\mathcal{C}$ the category of central simple algebras of degree $n$ with involution, and by $\mathcal{P}$ the category of irreducible $P_{n, \tau}$-varieties which are generically free as $\mathrm{PGL}_{n}$-varieties. By Lemmas 2.1 and 2.4(a), the assignment $\mathcal{F}$ in Theorem 1.1 is well-defined. By [10, Corollary 8.6(a)], $\mathcal{F}$ is a contravariant functor from $\mathcal{P}$ to $\mathcal{C}$. If $\varphi$ and $\alpha$ are as in Lemma 2.4, then $\left(\varphi^{*}\right)_{*}=\varphi$ and $\left(\alpha_{*}\right)^{*}=\alpha$, see [10, Proposition 8.4]. Hence $\mathcal{F}$ is full and faithful. Moreover, by Proposition 2.3, every object in $\mathcal{C}$ is isomorphic to the image of an object in $\mathcal{P}$. Hence $\mathcal{F}$ is a covariant equivalence of categories between $\mathcal{P}$ and the dual category of $\mathcal{C}$, see, e.g., [1, Theorem 7.6]. In other words, $\mathcal{F}$ is a contravariant equivalence of categories between $\mathcal{P}$ and $\mathcal{C}$, as claimed.

\section{Involutions of the First and Second Kind}

In this section, we prove a characterization of involutions of the first and second kind in terms of the generic freeness of the action of $P_{n, \tau}$ on the associated variety. As an example, we then consider the involution $\rho$ of $U D(m, n)$, which is of the first kind if and only if $m=n=2$.

Lemma 3.1. Let $X$ and $\sigma_{X, \tau}$ be as in Lemma 2.1. If $\sigma_{X, \tau}$ is an involution of the second kind, then the $P_{n, \tau}$-action on $X$ is generically free.

Note that the converse is also true, see Corollary 3.3 below.

Proof. Let $\sigma=\sigma_{X, \tau}$. Recall that the center of $k_{n}(X)$ is $k(X)^{\mathrm{PGL}_{n}}=k(Y)$, where $Y$ is any model of the rational quotient $X / \mathrm{PGL}_{n}$ for the action of $\mathrm{PGL}_{n}$ on $X$. Since $\mathrm{PGL}_{n}$ is a normal subgroup of $P_{n, \tau}$, the $P_{n, \tau}$-action on $k(X)$ induces an action of $\langle\tau\rangle \cong P_{n, \tau} / \mathrm{PGL}_{n}$ on $k(Y)$ as follows: For $f \in k(Y), \tau(f)=f \circ \tau^{-1}=f \circ \tau=\sigma(f)$, where the last equality holds since the image of $f$ consists of scalar matrices. Thus $k(Y)^{\langle\tau\rangle}$ is the fixed field of the center of $k_{n}(X)$ under the action of the involution $\sigma$. Since $\sigma$ is an involution of the second kind, $k(Y)$ is thus a field extension of $k(Y)^{\langle\tau\rangle}$ of degree 2 .

We can chose $Y$ to be a model for the rational quotient $X / \mathrm{PGL}_{n}$ such that the action of $P_{n, \tau}$ on $X$ induces a regular action of $\langle\tau\rangle \cong P_{n, \tau} / \mathrm{PGL}_{n}$ on $Y$, cf. [6, Proposition 2.6 and Corollary to Theorem 1.1]. Denote by $\pi_{1}: X \rightarrow Y$ and $\pi_{2}: Y \rightarrow Y /\langle\tau\rangle$ the rational quotient maps for the actions of $\mathrm{PGL}_{n}$ and $\langle\tau\rangle$, respectively. Then $Y /\langle\tau\rangle$ is a rational quotient for the $P_{n, \tau}$-action on $X$, with quotient map $\pi_{2} \circ \pi_{1}$. 
Because $k(Y)$ is a field extension of $k(Y)^{\langle\tau\rangle}$ degree 2, the fibers in general position of $\pi_{2}$ consist of two points. The fibers in general position of $\pi_{1}$ consist of the distinct $\mathrm{PGL}_{n}$-orbits in $X$. Thus the fibers in general position of the quotient map $\pi_{2} \circ \pi_{1}$ for the rational quotient for the $P_{n, \tau}$ action on $X$ consist of two distinct $\mathrm{PGL}_{n}$-orbits. Since the $\mathrm{PGL}_{n}$-action on $X$ is generically free, and since $\mathrm{PGL}_{n}$ has index 2 in $P_{n, \tau}$, it follows that the $P_{n, \tau}$-action on $X$ is generically free.

Corollary 3.2. Consider the involution $\sigma_{\tau, X}$ in Lemma 2.1. The following are equivalent:

(1) $\sigma_{\tau, X}$ is an involution of the first kind.

(2) The $P_{n, \tau}$-orbits in $X$ in general position are $\mathrm{PGL}_{n}$-orbits.

(3) The $P_{n, \tau}$-orbits in $X$ in general position are irreducible.

(4) The $P_{n, \tau}$-action on $X$ is not generically free.

Proof. Let $\sigma=\sigma_{\tau, X}$. By definition, $\sigma$ is an involution of the first kind iff $\sigma(f)=f$ for all $f \in \mathrm{Z}(A)=k(X)^{\mathrm{PGL}_{n}}$.

$(1) \Longrightarrow(2)$ : For $f \in k(X)^{\mathrm{PGL}_{n}}$, and for $x \in X$ in general position,

$$
f(x)=\sigma(f)(x)=f(\tau x)^{t}=f(\tau x),
$$

where the last equality follows from $f \in k(X)^{\mathrm{PGL}_{n}}$. Since finitely many $f \in$ $k(X)^{\mathrm{PGL}_{n}}$ separate the $\mathrm{PGL}_{n}$-orbits in $X$ in general position, this implies that $\tau(x)$ belongs to the $\mathrm{PGL}_{n}$-orbit of $x$. Hence the $P_{n, \tau}$-orbits in general position in $X$ are just the $\mathrm{PGL}_{n}$-orbits.

$(2) \Longrightarrow(3)$ : Clear since $\mathrm{PGL}_{n}$ is connected.

(3) $\Longrightarrow(4)$ : Clear since $P_{n, \tau}$ is not connected.

$(4) \Longrightarrow(1)$ : By Lemma 3.1, $\sigma$ cannot be of the second kind.

Corollary 3.3. Let $A$ be a central simple algebra of degree $n$ with involution $\sigma$. Let $X$ be the associated $P_{n, \tau}$-variety. Then $\sigma$ is an involution of the first kind if and only if the action of $P_{n, \tau}$ on $X$ is not generically free. Equivalently, $\sigma$ is an involution of the second kind if and only if the action of $P_{n, \tau}$ on $X$ is generically free.

Proof. By Proposition 2.3, we may assume that $(A, \sigma)=\left(k_{n}(X), \sigma_{\tau, X}\right)$, where $\sigma_{\tau, X}$ is as in Lemma 2.1. Now the result immediately follows from Corollary 3.2 .

Remark 3.4. One can use a theorem of Richardson's to somewhat simplify the proofs in this section (though not in Section 5). Let $X$ be an irreducible $P_{n, \tau}$-variety which is $\mathrm{PGL}_{n}$-generically free. Since $\mathrm{PGL}_{n}$ has index 2 in $P_{n, \tau}$, it follows that for $x \in X$ in general position, the stabilizer of $x$ in $P_{n, \tau}$ has at most 2 elements. In particular, these stabilizers are reductive. Hence by a theorem of Richardson [12, Theorem 9.3.1], there is a stabilizer in general position for the $P_{n, \tau}$-action on $X$. Using this fact, one can now obtain Lemma 3.1 as an immediate consequence of Corollary 3.2, if one modifies the proof of the latter result as follows: 
$(2) \Longrightarrow(1)$ : Let $f \in k(X)^{\mathrm{PGL}_{n}}$. If $x \in X$ is in general position, there is an $h \in \mathrm{PGL}_{n}$ such that $\tau(x)=h(x)$. Then $\sigma(f)(x)=f(\tau x)^{t}=f(\tau x)=$ $f(h x)=f(x)$. Hence $\sigma(f)=f$.

$(4) \Longrightarrow(2)$ : If a stabilizer in general position exists, it cannot be the trivial subgroup. Since the $\mathrm{PGL}_{n}$-action on $X$ is generically free, for $x \in X$ in general position, there is thus a $g \in P_{n, \tau} \backslash \mathrm{PGL}_{n}$ such that $g x=x$. Since $P_{n, \tau}=\mathrm{PGL}_{n} \cup \mathrm{PGL}_{n} g$, the $P_{n, \tau}$-orbit of $x$ is equal to the $\mathrm{PGL}_{n}$-orbit of $x$.

Example 3.5. The involution $\rho$ of $U D(m, n)$ (see Example 2.9) is of the first kind if and only if $m=n=2$. For $n \geq 3$, this follows from [7, Proposition 7.2], which states that the restriction of $\rho$ to the center of $U D(m, n)$ cannot be induced by an automorphism of the corresponding trace ring, so in particular cannot be the identity map. Also the case $n=2$ can be handled with the methods of $[7]$. For the convenience of the reader, we include the short arguments.

Note that the (reduced) trace on $U D(m, n)$ is $\rho$-equivariant, see [3, Corollaries 2.2 and 2.16]. Assume first that $m=n=2$. Denote the two generic matrices generating $U D(2,2)$ by $X$ and $Y$. Then the center of $U D(2,2)$ is generated by $\operatorname{tr}(X), \operatorname{tr}(Y), \operatorname{tr}\left(X^{2}\right), \operatorname{tr}\left(Y^{2}\right)$ and $\operatorname{tr}(X Y)$. Since $\rho$ fixes $X, Y, X^{2}$ and $Y^{2}$, it fixes the traces of these elements. And $\rho(\operatorname{tr}(X Y))=\operatorname{tr}(\rho(X Y))=\operatorname{tr}(Y X)=\operatorname{tr}(X Y)$, showing that $\rho$ acts trivially on the center of $U D(2,2)$, so that $\rho$ is an involution of the first kind. We will see in Section 6 that $\rho$ is in this case an orthogonal involution.

Assume next that $m \geq 3$, and let $X, Y$ and $Z$ be three distinct generic matrices in $U D(m, n)$. Evaluating these generic matrices at the elementary matrix units $e_{1,2}, e_{2,2}$ and $e_{2,1}$, respectively, shows that $\operatorname{tr}(X Y Z) \neq \operatorname{tr}(Z Y X)$. Since $\operatorname{tr}(Z Y X)=\operatorname{tr}(\rho(X Y Z))=\rho(\operatorname{tr}(X Y Z))$, it follows that $\rho$ is of the second kind.

Finally, assume that $m=2$ and $n \geq 3$. Denote the two generic matrices generating $U D(m, n)$ by $X$ and $Y$. Evaluating $X$ and $Y$ at $e_{1,2}+e_{2,3}$ and $e_{1,2}+e_{3,1}$, respectively, shows that $\operatorname{tr}\left(X Y X^{2} Y^{2}\right) \neq \operatorname{tr}\left(Y^{2} X^{2} Y X\right)$. Consequently, $\rho$ is of the second kind.

Remark 3.6. Using Corollary 3.2, we can reinterpret this example as stating that that the action of $P_{n, \tau}$ on $\left(\mathrm{M}_{n}\right)^{m}$ is generically free if and only if $(m, n) \neq(2,2)$.

\section{Preliminaries on Transposition in $\mathrm{PGL}_{n}$}

Given $a \in \mathrm{GL}_{n}$, we denote by $\bar{a}$ its image in $\mathrm{PGL}_{n}$. Let $a, b \in \mathrm{GL}_{n}$ such that $\bar{a}=\bar{b}$, i.e., $b=\lambda a$ for some nonzero scalar $\lambda$. Then $b^{t}=\lambda a^{t}$. Hence we can define $\bar{a}^{t}=\bar{a}^{t}$. One easily checks that for $a, b \in \mathrm{GL}_{n},\left(\bar{a}^{t}\right)^{t}=\bar{a}$, that $(\bar{a} \bar{b})^{t}=\bar{b}^{t} \bar{a}^{t}$, and that $\left(\bar{a}^{-1}\right)^{t}=\left(\bar{a}^{t}\right)^{-1}$.

Now let $a \in \mathrm{GL}_{n}$ such that $\bar{a}=\bar{a}^{t}$. Then $a^{t}=\epsilon a$ for some nonzero scalar $\epsilon$. Denoting the $i, j$-entry of $a$ by $a_{i, j}$, it follows that $a_{i, j}=\epsilon a_{j, i}=\epsilon^{2} a_{i, j}$. Consequently $\epsilon= \pm 1$, i.e., $a$ is either a symmetric or a skew-symmetric 
matrix. If $b \in \mathrm{GL}_{n}$ with $\bar{b}=\bar{a}$, then $b^{t}=\epsilon b$. This allows us to make the following definition.

Definition 4.1. Let $g \in \mathrm{PGL}_{n}$ with $g^{t}=g$. We call $g$ symmetric (respectively, skew-symmetric) if one (and hence all) preimages of $g$ in $\mathrm{GL}_{n}$ are symmetric (respectively, skew-symmetric).

Remark 4.2. Let $a \in \mathrm{GL}_{n}$ such that $a^{t}=\epsilon a$ with $\epsilon= \pm 1$. Then $\operatorname{det}(a)=$ $\operatorname{det}\left(a^{t}\right)=\epsilon^{n} \operatorname{det}(a)$, implying $\epsilon^{n}=1$. Since $\epsilon= \pm 1$, this implies $\epsilon=1$ if $n$ is odd. So for $n$ odd, there are no skew-symmetric elements in $\mathrm{PGL}_{n}$. As we will see, this corresponds to the fact that involutions of the first kind on central simple algebras of odd degree cannot by symplectic, see [3, Corollary 2.8(1)].

Remark 4.3. For future use, we record a few technical facts. Again, let $a \in \mathrm{GL}_{n}$ such that $a^{t}=\epsilon a$ with $\epsilon= \pm 1$. Then $\left(a^{-1}\right)^{t}=\epsilon^{-1} a^{-1}=\epsilon a^{-1}$. Hence if $g \in \mathrm{PGL}_{n}$ is symmetric (respectively, skew-symmetric) then so is $g^{-1}$. Recall that a matrix is congruent to $a$ if it is of the form $b a b^{t}$ for some $b \in \mathrm{GL}_{n}$. Note that $\left(b a b^{t}\right)^{t}=b a^{t} b^{t}=\epsilon\left(b a b^{t}\right)$. Consequently, if $g \in \mathrm{PGL}_{n}$ is symmetric (respectively, skew-symmetric), then so are $h g h^{t}$ and $h g^{-1} h^{t}$ for any $h \in \mathrm{PGL}_{n}$.

Remark 4.4. Let $g \in \mathrm{PGL}_{n}$ be symmetric or skew-symmetric. Given any $h \in \mathrm{PGL}_{n}, \tau g$ is conjugate in $P_{n, \tau}$ to $h^{-1}(\tau g) h=\tau\left(h^{t} g h\right)$. Recall that any non-singular symmetric matrix over an algebraically closed field of characteristic $\neq 2$ is congruent to the identity matrix, while any non-singular skew-symmetric matrix is congruent to

$$
J=\left(\begin{array}{cc}
0 & I \\
-I & 0
\end{array}\right),
$$

where $I$ is the identity matrix of order $\frac{n}{2}$ (see, e.g., [2], Sections 8.3 and 8.6]). Consequently, if $g$ is symmetric, $\tau g$ is conjugate in $P_{n, \tau}$ to $\tau$, while if $g$ is skew-symmetric, $\tau g$ is conjugate in $P_{n, \tau}$ to $\tau g_{0}$, where $g_{0}$ is the image in $\mathrm{PGL}_{n}$ of the matrix $J$.

\section{Proof of Theorem 1.2}

By Proposition 2.3, we may assume that $(A, \sigma)=\left(k_{n}(X), \sigma_{\tau, X}\right)$, where $\sigma_{\tau, X}$ is as in Lemma 2.1. Part (b) of Theorem 1.2 was already proved in Corollary 3.3. Note that part (a) will immediately follow from part (b), once we proved the existence of the stabilizer in general position.

If $\sigma$ is an involution of the second kind, we already saw that the action of $P_{n, \tau}$ on $X$ is generically free and thus has a stabilizer in general position, namely $S=\{1\}$.

Assume now that $\sigma$ is an involution of the first kind. Since the $\mathrm{PGL}_{n^{-}}$ action on $X$ is generically free, and since $\mathrm{PGL}_{n}$ has index 2 in $P_{n, \tau}$, Corollary 3.2 implies that every $x \in X$ in general position must have a stabilizer in $P_{n, \tau}$ of order 2 . The nontrivial element in the stabilizer must 
be of form $\tau g_{x}$ for some $g_{x} \in \mathrm{PGL}_{n}$ (depending on $x$ ). Note that then $\tau x=g_{x} x$. Hence $x=\tau^{2} x=\tau g_{x} x=\left(\left(g_{x}\right)^{-1}\right)^{t} \tau x=\left(\left(g_{x}\right)^{-1}\right)^{t} g_{x} x$. Since $\left(\left(g_{x}\right)^{-1}\right)^{t} g_{x}=\left(\left(g_{x}\right)^{t}\right)^{-1} g_{x}$ belongs to PGL $n$, it must be 1, i.e., $\left(g_{x}\right)^{t}=g_{x}$. Hence $g_{x}$ is either a symmetric or a skew-symmetric element of $\mathrm{PGL}_{n}$ (see Definition 4.1).

Let $F$ be the center of $A$. We denote by $\operatorname{Sym}(A, \sigma)$ and $\operatorname{Skew}(A, \sigma)$ the $F$ subspaces of symmetric and skew-symmetric elements of $A$ with respect to $\sigma$. Let $\left\{a_{i}\right\}$ and $\left\{b_{j}\right\}$ be $F$-bases of $\operatorname{Sym}(A, \sigma)$ and $\operatorname{Skew}(A, \sigma)$, respectively. For $x \in X$ in general position, $\left\{a_{i}(x)\right\}$ and $\left\{b_{j}(x)\right\}$ are $k$-linearly independent subsets of $\mathrm{M}_{n}$ by [8, Lemma 7.4(a)].

Denote by $U$ a dense open subset of $X$ such that all $x \in U$ have the following properties: $a_{i}(x)$ and $b_{j}(x)$ are defined for all $i$ and $j$, and the stabilizer of $x$ in $P_{n, \tau}$ has two elements (so that $g_{x}$ is defined).

Lemma 5.1. Let $x \in U$. Then $g_{x}$ is symmetric iff $\sigma$ is orthogonal.

Proof. For simplicity, set $g=g_{x}$. Let $u$ be a preimage of $g^{-1}$ in $\mathrm{GL}_{n}$. So $u^{t}= \pm u$. Denote by $\operatorname{Int}(u)$ conjugation by $u$ on $\mathrm{M}_{n}$. Transposition defines an involution of orthogonal type of $\mathrm{M}_{n}$. By [3, Proposition 2.7(1)],

$$
\tilde{\sigma}=\operatorname{Int}(u) \circ \text { transposition }
$$

is an involution of $\mathrm{M}_{n}$ of the first kind. Moreover, by [3, Proposition 2.7(3)], $\tilde{\sigma}$ is of the same type as transposition (i.e., is of orthogonal type) iff $u^{t}=u$. Consequently, $\tilde{\sigma}$ is of orthogonal type iff $u^{t}=u$ iff $g^{-1}$ is symmetric iff $g$ is symmetric.

Let $f$ be any element of $A$ defined at $x$. Using the definition of $\sigma$ (see Lemma 2.1) and the facts that $f$ is $\mathrm{PGL}_{n}$-equivariant and that $u^{t}= \pm u$, we see that

$$
\begin{aligned}
\sigma(f)(x) & =f(\tau x)^{t}=f(g x)^{t}=(g \cdot f(x))^{t}=\left(u^{-1} f(x) u\right)^{t} \\
& =u^{t} f(x)^{t}\left(u^{-1}\right)^{t}=u f(x)^{t} u^{-1}=\tilde{\sigma}(f(x)) .
\end{aligned}
$$

Hence $a_{i}(x)=\sigma\left(a_{i}\right)(x)=\tilde{\sigma}\left(a_{i}(x)\right)$, and $-b_{j}(x)=\sigma\left(b_{j}\right)(x)=\tilde{\sigma}\left(b_{j}(x)\right)$. Thus $\operatorname{dim}_{k}\left(\operatorname{Sym}\left(\mathrm{M}_{n}, \tilde{\sigma}\right)\right) \geq \operatorname{dim}_{F}(\operatorname{Sym}(A, \sigma))$, and $\operatorname{dim}_{k}\left(\operatorname{Skew}\left(\mathrm{M}_{n}, \tilde{\sigma}\right)\right) \geq$ $\operatorname{dim}_{F}(\operatorname{Skew}(A, \sigma))$. It follows by [3, Proposition 2.6(1)] that $\sigma$ and $\tilde{\sigma}$ are of the same type. Hence $\sigma$ is of orthogonal type iff $\tilde{\sigma}$ is of orthogonal type iff $g$ is symmetric.

Lemma 5.1 has several immediate consequences. First, for $x \in U, g_{x}$ is skew-symmetric iff $\sigma$ is symplectic. Secondly, either $g_{x}$ is symmetric for all $x \in U$, or $g_{x}$ is skew-symmetric for all $x \in U$.

Now let $x, y \in U$. So $g_{x}$ and $g_{y}$ are either both symmetric or both skewsymmetric. Hence there is an $h \in \mathrm{PGL}_{n}$ such that $g_{y}=h^{t} g_{x} h$, cf. Remark 4.4. Consequently, $\tau g_{y}=\tau\left(h^{t} g_{x} h\right)=h^{-1}\left(\tau g_{x}\right) h$ is conjugate to $\tau g_{x}$ in $P_{n, \tau}$. Since the stabilizers of $x$ and $y$ in $P_{n, \tau}$ are $\left\{1, \tau g_{x}\right\}$ and $\left\{1, \tau g_{y}\right\}$, respectively, they are conjugate in $P_{n, \tau}$. Since this is true for all $x$ and $y$ in the dense open subset $U$ of $X$, it follows that there is a stabilizer in general 
position for the $P_{n, \tau}$-action on $X$. Moreover, by Remark 4.4, the stabilizer in general position will be conjugate to $\{1, \tau\}$ if $\sigma$ is orthogonal, and to $\left\{1, \tau g_{0}\right\}$ if $\sigma$ is symplectic. This completes the proof of Theorem 1.2.

\section{The Involution $\rho$ of $U D(2,2)$}

We now illustrate Theorem 1.2 in the case of the universal division algebra $U D(2,2)$. Recall from Example 2.2 that the $P_{n, \tau}$-variety associated to $(U D(m, n), \rho)$ is $\left(\mathrm{M}_{n}\right)^{m}$, where $\mathrm{PGL}_{n}$ acts by component-wise conjugation and $\tau$ acts by component-wise transposition. We observed in Example 3.5 that the involution $\rho$ of $A=U D(2,2)$ is an involution of the first kind, and that it fixes both of the generic matrices generating $A$. Hence $\operatorname{dim} \operatorname{Sym}(A, \rho) \geq 2$. Since $\operatorname{deg} A=n=2$, [3, Proposition 2.6(1)] implies that $\rho$ is of orthogonal type. Hence by Theorem 1.2(c), the action of $\tau$ on $\left(\mathrm{M}_{2}\right)^{2}$ is of symmetric type (using the terminology of Remark 1.3). We now verify this directly.

Let $x=\left(x_{1}, x_{2}\right) \in\left(\mathrm{M}_{2}\right)^{2}$ be in general position. We may assume that $x_{1}$ is diagonalizable, and that $\mathrm{M}_{2}=k\left\{x_{1}, x_{2}\right\}$. Hence there is an $h \in \mathrm{PGL}_{2}$ such that $y=h \cdot x$ is of form

$$
y=\left(y_{1}, y_{2}\right)=\left(\left(\begin{array}{cc}
\lambda & 0 \\
0 & \mu
\end{array}\right),\left(\begin{array}{ll}
a & b \\
c & d
\end{array}\right)\right),
$$

with $\lambda, \mu, a, b, c, d \in k$. Since $\mathrm{M}_{2}=k\left\{x_{1}, x_{2}\right\}=k\left\{y_{1}, y_{2}\right\}$, both $b, c \neq 0$. Denote by $g$ the image in $\mathrm{PGL}_{2}$ of the diagonal matrix $\left(\begin{array}{ll}c & 0 \\ 0 & b\end{array}\right)$. Note that $g$ is symmetric according to Definition 4.1. A short calculation shows that $g \cdot y=y^{t}$. Hence $(g h) \cdot x=g \cdot y=y^{t}=(h \cdot x)^{t}=\left(h^{-1}\right)^{t} \cdot x^{t}$, so that $\tau \cdot x=x^{t}=\left(h^{t} g h\right) \cdot x$. As we saw in Remark 4.3, $h^{t} g h$ is symmetric since $g$ is. It follows that the action of $\tau$ on $\left(\mathrm{M}_{2}\right)^{2}$ is of symmetric type.

\section{Models for Associated Varieties}

Up to birational isomorphism, the associated variety of a central simple algebra can always be found in $\left(\mathrm{M}_{n}\right)^{m}$ for some $m \geq 2$, where $\mathrm{PGL}_{n}$ acts on $\left(\mathrm{M}_{n}\right)^{m}$ by simultaneous conjugation. To be more precise, denote by $U_{m, n}$ the subset of $\left(\mathrm{M}_{n}\right)^{m}$ consisting of all tuples $\left(a_{1}, \ldots, a_{m}\right)$ in $\left(\mathrm{M}_{n}\right)^{m}$ such that $a_{1}, \ldots, a_{m}$ generate $\mathrm{M}_{n}$ as $k$-algebra. An $n$-variety is a closed $\mathrm{PGL}_{n}$-invariant subvariety of $U_{m, n}$ for some $m \geq 2$. It is shown in 10 , Lemma 8.1] that every irreducible generically free $\mathrm{PGL}_{n}$-variety is $\mathrm{PGL}_{n^{-}}$ equivariantly birationally isomorphic to an irreducible $n$-variety in $U_{m, n}$ for some $m$. Adapting the proof of this lemma, we now obtain the corresponding result for the associated varieties of central simple algebras with involution.

Lemma 7.1. Let $X$ be an irreducible $P_{n, \tau}$-variety which is generically free as $\mathrm{PGL}_{n}$-variety. If the action of $P_{n, \tau}$ is of skew-symmetric type, assume additionally that $n>2$. There is an irreducible $n$-variety $Y$ in $\left(\mathrm{M}_{n}\right)^{m}$ for some $m \geq 2$ which is invariant under component-wise transposition (and thus a $P_{n, \tau}$-variety) such that $X$ is $P_{n, \tau}$-equivariantly isomorphic to $Y$. 
Here $P_{n, \tau}$ acts on $\left(\mathrm{M}_{n}\right)^{m}$ and $Y$ as in Example 2.2. If the action of $P_{n, \tau}$ is of skew-symmetric type (see Remark 1.3), the result is not true in case $n=2$ : an easy calculation shows that $U_{2, m}$ does not contain points whose stabilizer in $P_{n, \tau}$ is $\left\langle\tau\left[\begin{array}{cc}0 & 1 \\ -1 & 0\end{array}\right]\right\rangle$.

Proof. The $P_{n, \tau}$-action on $X$ has a stabilizer $H$ in general position as in Theorem 1.2. Assume first that $H=1$. Then the proof of [10, Lemma 8.1] goes through with minor changes, if one replaces $\mathrm{PGL}_{n}$ by $P_{n, \tau}$ throughout: By Remark 3.6, there is a dense open subset $V$ of $\left(\mathrm{M}_{n}\right)^{3}$ such that every element of $V$ has trivial stabilizer in $P_{n, \tau}$. Now set $m=r+3$, and construct a $P_{n, \tau}$-equivariant rational map $\phi: X \rightarrow\left(\mathrm{M}_{n}\right)^{3}$ whose image meets $U_{3, n} \cap V$; the latter enables us to choose the subset $S \subset X$ in such a way that all points of $f(S)$ have trivial stabilizer in $P_{n, \tau}$.

Now assume that $H=\langle\tau\rangle$ or that $H=\left\langle\tau g_{0}\right\rangle$ where $g_{0}=\left[\begin{array}{cc}0 & I \\ -I & 0\end{array}\right]$. Since both $P_{n, \tau}$ and $H$ are reductive, $X$ is $P_{n, \tau}$-equivariantly birationally isomorphic to a stable affine $P_{n, \tau}$-variety, see [9, Theorem 1.1]. Hence we may assume that $X$ is affine, and that the $P_{n, \tau}$-action on $X$ is stable. Pick $x_{0} \in X$ with stabilizer $H$ and such that the $P_{n, \tau}$-orbit of $x_{0}$ is closed. For some $q$, we can find $a_{0} \in U_{q, n} \subset\left(\mathrm{M}_{n}\right)^{q}$ such that also $a_{0}$ has stabilizer $H$ : If $H=\langle\tau\rangle$, this is clear since $\mathrm{M}_{n}$ can be generated as $k$-algebra by $q$ symmetric matrices for some $q$. And an easy computation deals with the case $n \geq 4$ and $H=\left\langle\tau g_{0}\right\rangle$. It follows by [11, Lemma 2.6] (a slight extension of [5, Theorem 1.7.12]) that there is a $P_{n, \tau^{-}}$equivariant morphism $\phi: X \rightarrow\left(\mathrm{M}_{n}\right)^{q}$ with $\phi\left(x_{0}\right)=a_{0}$.

It follows from the proof of Lemma 3.1 (or from Corollary 3.2(b) and [8, Remark 2.5]), that under the current hypotheses, $X / P_{n, \tau}=X / \mathrm{PGL}_{n}$, so that $k(X)^{P_{n, \tau}}=k(X)^{\mathrm{PGL}_{n}}$.

Using the map $\phi$ we just constructed and setting $m=r+q$, the remainder of the proof of [10, Lemma 8.1] goes through nearly literally. Note that since $k(X)^{\mathrm{PGL}_{n}}=k(X)^{P_{n, \tau}}, f$ is $P_{n, \tau}$-equivariant.

\section{REFERENCES}

[1] T. S. Blyth, Categories, Longman, London and New York, 1986.

[2] P. M. Cohn, Algebra, Volume 1, second edition, John Wiley \& Sons, Chichester, 1982.

[3] M.-A. Knus, A. Merkurjev, M. Rost and J.-P. Tignol, The Book of Involutions, American Mathematical Society, Providence, RI, 1998.

[4] V. E. Kordonskii, On the birational classification of algebraic group actions, Izv. Ross. Akad. Nauk Ser. Mat. 65 (2001), 61-76; English translation in Izv. Math. 65 (2001), 57-70.

[5] Popov, V. L., Sections in invariant theory, The Sophus Lie Memorial Conference (Oslo, 1992), Scand. Univ. Press, Olso, 1994, 315-361.

[6] V. L. Popov and E. B. Vinberg, Invariant Theory, Algebraic Geometry IV, Encyclopedia of Mathematical Sciences 55, Springer, 1994, 123-284.

[7] Z. Reichstein, On automorphisms of matrix invariants induced from the trace ring, Linear Algebra Appl. 193 (1993), 51-74.

[8] Z. Reichstein, On the notion of essential dimension for algebraic groups, Transform. Groups 5 (2000), no. 3, 265-304. 
[9] Z. Reichstein and N. Vonessen, Stable affine models for algebraic group actions, J. Lie Theory 14 (2004), 563-568.

[10] Z. Reichstein and N. Vonessen, Polynomial identity rings as rings of functions, J. Algebra 310 (2007), 624-647.

[11] Z. Reichstein and N. Vonessen, Group actions on central simple algebras: A geometric approach, J. Algebra 304 (2006), 1160-1192.

[12] R. W. Richardson Jr., Deformations of Lie subgroups and the variation of isotropy subgroups, Acta Math. 129 (1972), 35-73.

[13] L. Rowen, Ring Theory, Volume I, Academic Press, San Diego, 1988.

Department of Mathematical Sciences, University of Montana, Missoula, MT 59812-0864, USA

E-mail address: hikolaus.vonessen@umontana.edu

$U R L$ : www.math.umt.edu/vonessen 